\title{
Efeito do omeprazol e do pantoprazol sobre a regeneração hepática após hepatectomia parcial em ratos ${ }^{1}$
}

\author{
Gustavo Barreto de Melo ${ }^{2}$ \\ Renata Lemos Silva ${ }^{3}$ \\ Ricardo Fakhouri ${ }^{4}$ \\ Valdinaldo Aragão de Melo 5 \\ Sônia Oliveira Lima ${ }^{6}$
}

\begin{abstract}
Melo GB, Silva RL, Fakhouri R, Melo VA, Lima SO. Efeito do omeprazol e do pantoprazol sobre a regeneração hepática após hepatectomia parcial em ratos. Acta Cir Bras [serial online] 2003 Nov-Dez;18(6). Disponível em URL: http://www.scielo.br/acb.

RESUMO - Objetivo: Avaliar os efeitos do omeprazol e do pantoprazol sobre a regeneração hepática após hepatectomia parcial. Métodos: Cinqüenta e oito ratos Wistar machos foram divididos em 4 grupos: Grupo SHAM, Grupo HP, Grupo PANTO e Grupo OMEP. Eles foram submetidos a hepatectomia parcial de 67\% (Grupos HP, PANTO e OMEP) ou laparotomia (Grupo SHAM). Os fígados foram removidos 32 e 56 horas após a operação. Depois, os animais foram sacrificados. Em todos os grupos, as substâncias (solução salina, omeprazol e pantoprazol) foram aplicadas diariamente a partir do momento em que foram operados até o sacrifício. Resultados: O índice de mitose no Grupo SHAM não foi significativo. Trinta e duas horas após a hepatectomia, a contagem de mitoses foi de 1,2 $\pm 1,09$ para o Grupo HP, 1,2 \pm 1,6 para o Grupo OMEP e 2,6 \pm 3,2 para o Grupo PANTO. Na análise após 56 horas, os valores foram 1,6 \pm ,89 para o HP, $2 \pm 1,8$ para o OMEP e 2,6 \pm 0,54 para o PANTO. Esses resultados não foram estatisticamente significativos. Conclusão: O omeprazol e o pantoprazol, agentes inibidores da bomba de prótons (H+, K+-ATPase), não interferem na regeneração hepática 32 e 56 horas após hepatectomia parcial a $67 \%$ em ratos.
\end{abstract}

DESCRITORES - Omeprazol. Pantoprazol. Hepatectomia parcial. Regeneração hepática.

\section{Introdução}

O hepatócito é uma célula que, em condições normais, raramente se divide. Entretanto, sua capacidade regenerativa se mantém extremamente elevada ${ }^{1}$.

Há várias condições que causam alteração da massa hepática. Ressecções para retirada de cistos ou tumores, lesões por produtos químicos ou vírus e transplantes são ocasiões que podem desencadear a replicação dos hepatócitos que estavam quiescentes ${ }^{1}$.

Na presença do estímulo à regeneração hepática, vários fatores diferentes interagem, criando uma complexa rede replicativa ${ }^{2}$. Assim, é importante ter conhecimento de tudo que possa influenciar esse processo.
Após grandes ressecções hepáticas, é comum a ocorrência de sangramento decorrente de úlceras causadas por estresse pós-operatório. Várias drogas, entre elas omeprazol e pantoprazol, são usadas para tratar essas úlceras e, profilaticamente, para evitar sua formação $0^{3,4}$.

Alguns estudos mostraram que o omeprazol, a depender da concentração, tanto pode inibir quanto estimular a regeneração do fígado ${ }^{5,6}$. Quanto ao efeito do pantoprazol, nenhum estudo foi encontrado. Assim, este trabalho propôsse a estudar os efeitos dessas drogas inibidoras da bomba de prótons gástrica (H+, K+-ATPase) sobre a regeneração hepática após hepatectomia parcial de $67 \%$.

\section{Métodos}

Este trabalho foi realizado de acordo com os preceitos do CIOMS (Council for International Organization of Medical Sciences) ethical code for animal experimentation e do Colégio Brasileiro de Experimentação Animal - COBEA.

Foram utilizados 58 ratos da raça Wistar, machos, pesando entre 160 e $250 \mathrm{~g}$ Os animais foram mantidos em biotério, com ciclo de claro-escuro natural, temperatura e umidades próprios do ambiente e foram alimentados com ração e água $a d$ libitum.

Os animais foram divididos em 4 grupos, sendo que metade de cada grupo foi avaliada após 32 horas e a outra, após 56 horas:

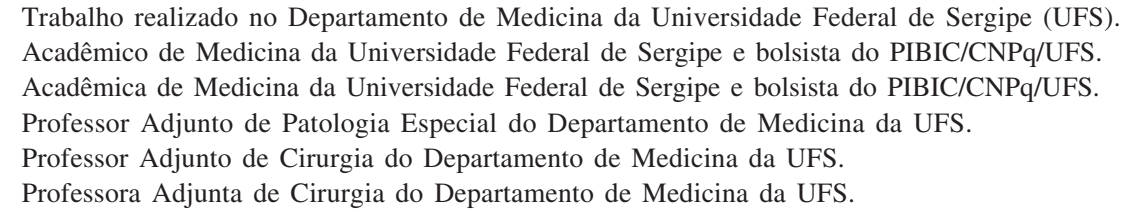


Grupo SHAM: composto por 10 animais que foram laparotomizados, tendo o fígado manipulado e recebendo injeção intramuscular de solução salina.

Grupo HP (controle): constituído por 16 animais submetidos à hepatectomia parcial e administração intramuscular de solução salina.

Grupo PANTO (pantoprazol): constituído por 16 animais submetidos à hepatectomia parcial e administração de $0,2 \mathrm{mg} /$ $\mathrm{kg}$ de pantoprazol por via intramuscular.

Grupo OMEP (omeprazol): também composto por 16 ratos, submetidos à hepatectomia parcial e administração intramuscular de $0,2 \mathrm{mg} / \mathrm{kg}$ de omeprazol.

Os procedimentos cirúrgicos foram realizados sempre no mesmo horário, para evitar influência do ciclo circadiano. $\mathrm{O}$ anestésico utilizado foi o éter etílico devido a sua praticidade e pelo fato de não possuir potencial hepatotóxico, como gases anestésicos halogenados, e não induzir o metabolismo de drogas ao nível do sistema microssomial hepático como os barbitúri$\cos ^{7}$. Usou-se polivilpirrolidona-iodo na anti-sepsia da parede abdominal após sua tricotomia.

A injeção de solução salina e das substâncias em estudo foi realizada logo após a indução anestésica, antes do início da cirurgia e por via intramuscular, na face posterior dos membros inferiores. As injeções subsequientes foram feitas alternadamente nos membros direito e esquerdo a cada 24 horas.

$\mathrm{O}$ acesso à cavidade peritoneal foi feito a partir de laparotomia mediana de cerca de $4 \mathrm{~cm}$ do apêndice xifóide em direção caudal. Em seguida, realizou-se a hepatectomia parcial, ressecando-se os lobos lateral esquerdo e mediano e fazendo ligadura de seus pedículos com fio de algodão. Essa ressecção equivale a aproximadamente $67 \%$ do volume do fígado ${ }^{8}$. A parede abdominal foi fechada com suturas contínuas, deixando o peritônio e a aponeurose num plano profundo e a pele, num plano superficial.

Os lobos hepáticos ressecados foram cortados em fragmentos homogêneos e fixados em formalina tamponada a $10 \%$. Depois, foram encaminhados para estudo histopatológico, corando-se os cortes histológicos pela hematoxilina e eosina. Foram analisados 10 campos ao microscópio óptico das regiões centrolobulares.
Os animais foram submetidos a nova laparotomia para retirada do remanescente hepático. Metade dos que compunham cada grupo foi operada ao término de 32 horas, enquanto a outra, 56 horas depois da cirurgia. Utilizou-se a mesma técnica anestésica para isso. Posteriormente, foram sacrificados.

O estudo dos dados foi realizado com o software de análise estatística SPSS 8.0. Os resultados obtidos foram analisados através do teste Mann-Whitney para comparação dos grupos, sendo considerados significativos os valores inferiores a $5 \%$.

\section{Resultados}

Na análise mitótica, o Grupo SHAM, que foi apenas laparotomizado, apresentou raros hepatócitos em mitose. Em relação aos grupos tratados com solução salina, omeprazol e pantoprazol, os resultados não apresentaram diferença estatisticamente significativa (Tabela 1 ).

Histologicamente, a arquitetura lobular e cordonal hepática estava preservada. Não foram identificadas alterações significativas dos espaços porta, exibindo regularidade dos seus contornos e integridade da placa limitante. Os hepatócitos exibiam discreta a moderada degeneração esteatótica do tipo microgoticular (Figura 1), fenômeno este observado em igual intensidade nos três grupos hepatectomizados. Foram observadas ainda, com relativa freqüência, células em apoptose nos cortes

TABELA 1 - Índice mitótico apresentado sob a forma de média e desvio-padrão

\begin{tabular}{c|ccc}
\multicolumn{1}{c}{ HP } & OMEP & PANTO \\
\hline 32 horas & $1,2 \pm 1,09$ & $1,2 \pm 1,6^{*}$ & $2,6 \pm 3,2^{*}$ \\
56 horas & $1,6 \pm 0,89$ & $2 \pm 1,8^{*}$ & $2,6 \pm 0,54^{*}$ \\
\hline
\end{tabular}

$\mathrm{p}>0,05$ vs HP

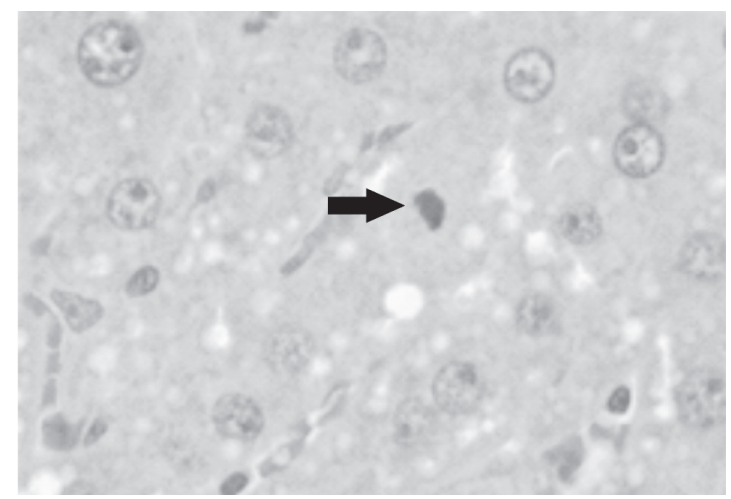

FIGURA 1 - Hepatócitos exibindo esteatose microgoticular. A seta indica célula em apoptose no Grupo HP (HE, 400X). de fígado dos animais do Grupo HP (Figura 1). Os sinusóides hepáticos apresentavam-se habituais.

\section{Discussão}

O fígado, quando lesado por doenças ou procedimentos cirúrgicos, tem grande capacidade de crescimento por hipertrofia e hiperplasia compensatória dos lobos remanescentes. Segundo Bucher (1967) ${ }^{9}$, o número de células em mitose nesse órgão aumenta aproximadamente 600 vezes, 24 horas após hepatectomia parcial de $67 \%$ no rato.

Os hepatócitos são o principal alvo de estudo da regeneração, já que constituem $90 \%$ da massa hepática e $60 \%$ do número total de células ${ }^{10}$. Vários fatores influenciam sua replicação. Alguns, considerados mitógenos completos, isoladamente, são suficientes para causar resposta regenerativa. Entre eles, estão o fator de crescimento do hepatócito (HGF), fator de crescimento epidérmico (EGF), fator transformador de crescimento - alfa (aTGF) e fator de crescimento de fibroblastos ácido (aFGF) ${ }^{2}$.

Entre os co-mitogênicos, que estimulam indiretamente, estão: substância estimuladora hepática (HSS), norepinefrina, vasopressina, angiotensinas II e III, insulina e glucagon, estrógeno e testosterona, prostaglandinas, fator de necrose tumoral - alfa (aTNF) e interleucina 6 (IL-6) ${ }^{2}$.

Ainda existem os inibidores, que regulam o fim da regeneração do fígado. $\mathrm{O}$ fator transformador de crescimento - beta (ßTGF), ativina $\mathrm{A}$ e interleucina 1 (IL-1) são alguns deles ${ }^{2}$.

Considerando a importância da regeneração hepática para a recuperação do fígado após hepatectomia, estudamos os efeitos do omeprazol e do pantoprazol. Ambas são inibidoras da bomba de prótons (H+, K+-ATPase) e atuam na redução da secreção gástrica ${ }^{4}$. Por isso, são bastante usadas profilaticamente no período pós-operatório de ressecções hepáticas para aliviar o stress cirúrgico e evitar a formação de úlceras e, conseqüentemente, sangramento ${ }^{5}$. 
Em trabalhos anteriores ${ }^{5,6}$, o omeprazol, aplicado durante 3 dias após a hepatectomia, estimulou a regeneração hepática com $0,2 \mathrm{mg} / \mathrm{Kg}$ ao dia e a inibiu com 0,4 $\mathrm{mg} / \mathrm{Kg}$ ao dia. Uma explicação exata para esses valores não foi encontrada.

Sabe-se que a estrutura do omeprazol, assim como do pantoprazol, é composta por anéis benzimidazólicos substituídos. Os anéis imidazólicos, dos quais são derivados, são inibidores do processo oxidativo nos microssomos hepáticos e, conseqüentemente, da regeneração do fígado ${ }^{6}$. Um estudo com hepatócitos in vitro mostrou que o omeprazol atua como um inibidor das mono-oxigenases do citocromo P-450 ${ }^{11}$. Já Francavilla et al $(1989)^{12}$ observaram que, nas mesmas condições de estudo, não afetou a proliferação dos hepatócitos e a estimulou após administrações repetidas da substância.

Além do efeito direto sobre os microssomos hepáticos, o omeprazol e o pantoprazol atuam de forma indireta. Isso ocorre já que ambas as substâncias causam aumento da secreção de gastrina, que é um fator hepatotrófico ${ }^{4,6,13}$.

No presente estudo, apesar de não terem sido feitas dosagens da gastrina e das enzimas hepáticas, acredita-se que os diferentes mecanismos de ação dos anéis benzimidazólicos substituídos do omeprazol e do pantoprazol tiveram seus efeitos compensados. Ou seja, enquanto a elevação da gastrina estimula a regeneração, os microssomos hepáticos são parcialmente inibidos. Isso pode, provavelmente, explicar por que, neste estudo, o omeprazol e o pantoprazol não apresentaram alterações sobre a divisão dos hepatócitos.

Um achado curioso observado neste estudo foi o freqüente número de células em apoptose nos fígados do grupo controle. A apoptose é um fenômeno muito importante em diversos aspectos da fisiologia celular, principalmente nos eventos de proliferação celular, dentre eles os processos de regeneração ${ }^{14,15}$. O menor número de apoptose nos dois grupos com medicação poderia traduzir um possível efeito protetor sobre os hepatócitos ou mesmo significar uma inativação dos sistemas indutores de apoptose desencadeada pelas drogas utilizadas. O significado deste achado é incerto e necessita de investigação mais aprofundada.

\section{Conclusão}

A administração de omeprazol e pantoprazol, agentes inibidores da bomba de prótons (H+, K+-ATPase), não revelou alteração sobre a regeneração hepática em 32 e em 56 horas após a hepatectomia, com uma concentração de $0,2 \mathrm{mg} / \mathrm{Kg}$ ao dia.

\section{Referências}

1. Fausto N. Liver regeneration. J Hepatol 2000; 32(1):19-31.

2. Ramalho FS. Regeneração hepática [Monografia]. Faculdade de Medicina de Ribeirão Preto da Universidade de São Paulo; 2000.

3. Lewis JH. Hepatic effects of drugs used in the treatment of peptic ulcer disease. Am J Gastroenterol 1987; 82:987-1003.
4. Jungnickel PW. Pantoprazole: a new proton pump inhibitor. Clin Ther 2000 22(11):1268-93

5. Aono T. The liver regenerative response elicited by antisecretory agents in partially hepatectomized rats: a comparison between omeprazole and famotidine. Surg Today 1995; 25(9):816-21.

6. Ohtake M, Aono T, Sakaguchi T, Tsukada $\mathrm{K}$, Hatakeyama K. Liver regeneration is enhanced by omeprazole in rats following partial hepatectomy. Br J Surg 1994; 81(8):1179-80.

7. Bertelli MSB. Toxicidade do acetominofen em ratos submetidos a hepatectomia parcial [Dissertação - Mestrado]. Fundação Faculdade Federal de Ciências Médicas de Porto Alegre; 1993.

8. Higgins GM, Anderson RM. Experimental pathology of the liver: restoration of liver of the white rat following partial surgical removal. Arch Pathol 1931; 12:186-202.

9. Bucher NLR. Experimental aspects of hepatic regeneration. N Engl J Med 1967; 13:686.

10. Alison MR. Regulation of hepatic growth Physiol Res 1986; 66:499-541.

11. Jensen JC, Gugler R. Inhibition of human liver cytochrome P-450 by omeprazole. Br J Clin Pharmacol 1986; 21:328-30.

12. Francavilla A, Panella C, Polimeno L, Di Leo A, Makowka L, Barone M, Amoruso A, Ingrosso M, Starzl TE. Effect of cimetidine, ranitidine, famotidine and omeprazole on hepatocyte proliferation in vitro. J Hepatol 1989; 8:32-41.

13. Rasmussen TN, Jørgensen PE, Almdal T, Poulsen SS, Olsen PS. Effect of gastrin on liver regeneration after partial hepatectomy in rats. Gut 1990; 31:92-5.

14. Kerr JF, Wyllie AH, Currie AR. Apoptosis: a basic biological phenomenon with wideranging implications in tissue kinetics. $\mathrm{Br} \mathbf{J}$ Cancer 1972; 26(4):239-57.

15. Carson DA, Ribeiro JM. Apopstosis and disease. Lancet 1993; 341(8855):1251-4.

\begin{abstract}
Melo GB, Silva RL, Fakhouri R, Melo VA, Lima SO. Effect of omeprazole and pantoprazole on liver regeneration after partial hepatectomy in rats. Acta Cir Bras [serial online] 2003 Nov-Dec;18(6). Available from URL: http://www.scielo.br/acb.

ABSTRACT - Purpose: To assess the effects of omeprazole and pantoprazole on liver regeneration after partial hepatectomy. Methods: Fifty eight male Wistar rats were divided into 4 groups: SHAM, HP, PANTO and OMEP Groups. They were submitted to 67\% partial hepatectomy (HP, PANTO and OMEP Groups) or laparotomy (SHAM Group). Their livers were removed 32 and 56 hours after the operation. Then, the animals were sacrificed. In all groups, the substances (saline solution, omeprazole and pantoprazole) were injected once daily from the moment they were operated on until the time of sacrifice. Results: In SHAM Group the mitotic index was not significant. Thirty two hours after hepatectomy, the mitosis index was 1.2 \pm 1.09 in HP Group, $1.2 \pm 1.6$ in OMEP Group and $2.6 \pm 3.2$ in PANTO Group. Concerning the 56-hour analysis, the values were $1.6 \pm 0.89$ in HP Group, $2 \pm 1.8$ in OMEP Group and $2.6 \pm 0.54$ in PANTO Group. These results were not statistically significant. Conclusion: Omeprazole and pantoprazole, proton pump inhibitors ( $\mathrm{H}+, \mathrm{K}+-\mathrm{ATPase})$, do not cause any change on liver regeneration 32 and 56 hours after $67 \%$ partial hepatectomy in rats.
\end{abstract}

KEY WORDS - Omeprazole. Pantoprazole. Partial hepatectomy. Liver regeneration.

Correspondência:

Prof. Dr. Valdinaldo Aragão de Melo

Rua Álvaro Brito, 14/301

49020-400 Aracaju - SE

vamelo@infonet.com.br
Conflito de interesse: nenhum Fontes de financiamento: PIBIC-CNPq/UFS

Data do recebimento: $14 / 08 / 2003$

Data da revisão: 01/09/2003

Data da aprovação: 21/09/2003 